\title{
Towards an electronic device to facilitate navigation in smartphones to people with motor and visual impairment
}

\author{
Silvia B. Fajardo-Flores, Laura S. Gaytán-Lugo, Gilberto Villagrana-Larios, \\ Pedro C. Santana Mancilla
}

Published: 30 November 2020

\begin{abstract}
In this paper, we analyze the physical characteristics of a user with both visual and motor impairment, as well as the user's context of smartphone use. In order to facilitate navigation using gestures that are easy to do by users with motor and visual impairment, we designed two hardware solutions as physical switches: a case mounted on the phone, and an electronic bracelet. We present the low fidelity prototype of both devices, the proposed user gestures, and their advantages and disadvantages.
\end{abstract}

\section{Keywords}

Wearable devices; Physical switches; Mobile accessibility; Motor impairment; Visual Impairment; Accessibility.

\section{Introduction}

This work is an extended version of the paper Electronic bracelet to facilitate navigation in smartphones to people with motor and visual impairment, by Fajardo-Flores, S. B., Gaytán-Lugo, L. S., Villagrana-Larios, G., \& Santana-Mancilla, P. C., presented at the IX Latin American Conference on Human Computer Interaction in 2019 [9].

People with physical disabilities are usually at disadvantage using smartphones. The integration of accessibility characteristics in mobile operating systems has significantly improved the users' interaction. Users with blindness use screen reader applications to navigate and to access contents, while users with motor impairment can adjust the configuration of the gestures. Accessibility features for users with visual or motor impairment are amongst the most useful, but current solutions for each impairment conflict with each other: A screen reader responds to gestures that a motor-impaired person might not be able to do, such as double-tapping or swiping. On the other hand, motor adjustments such as the tactile response time, requires that the user touches a specific position on the screen, which is not possible for people with blindness. Besides tapping, it is acknowledged that smartphone users with motor impairment

Fajardo-Flores, S. B., Santana-Mancilla, P. C.

School of Telematics

Universidad de Colima

medusa@ucol.mx,psantana@ucol.mx

Gaytán-Lugo, L. S., Villagrana-Larios G.

School of Mechanical and Electrical Engineering

Universidad de Colima

laura@ucol.mx,gvillagrana@ucol.mx have problems pressing physical buttons, and in some cases even holding the phone [16]. These difficulties make evident the need for alternative interactions.

In this work we focus on designing a solution to facilitate smartphone navigation to users with blindness and motor impairment. While blindness is the total loss of sight, a motor impairment is a condition that affects people in different ways: some people have weak limbs, some have deformities that hinder limb movements, others do not have limbs. It is worth mentioning that we made the design decisions considering the case of a user with blindness and a specific motor impairment, and that the proposed devices are targeted at people with similar characteristics.

\section{Related work}

\subsection{Accessible Mobile Interaction}

Providing access to technology resources is critical for people with disabilities because they need to perform tasks and use any application in the same way as other users [6]. Mobile devices play a central role in everyday lives of users around the world. According to Kane et al. [13] mobile devices create a feeling of safety and improve the ability to access information to people with disabilities.

However, mobile devices offer touchscreen interactions that may be particularly problematic for users with motor impairment [2]. Even more, for users who have both visual and motor disabilities.

Touchscreen accessibility is an active area of exploration. The screen reader solution for mobile devices allows people with visual impairment to read contents. The most common screen readers are VoiceOver for iOS devices and TalkBack for Android devices [8]. Screen readers respond to user interactions involving several gestures such as tapping and swiping with one or more fingers. However, these tasks are not always straightforward, and they usually take time [19].

Despite the accessibility benefits of touchscreens to people with motor disability, such as requiring a minimum amount of finger strength to register a tap, there are still many challenges: from simply pulling out the device to being able to tap precisely [10]. Likewise, different authors [7][11][14] argue that, although there have been advances in accessibility, mobile devices and applications still have issues, and they present important challenges for the use of people with visual impairment. 


\subsection{Assistive Technologies for People with Disabilities}

Assistive technologies (AT) refers to hardware and software designed to provide autonomy, social inclusion, and enhancement of quality of life to people with disabilities [18]. AT can be "low tech" or "high tech", from canes to conversational software [12].

Some of the most popular AT for people with visual and/or motor disability are the following:

Screen readers are applications of Text-To-Speech (TTS) synthesis. Screen readers are software used frequently by people with visual impairment to read text displayed on the computer screen with a speech synthesizer or braille display.

Braille terminal is an electromechanical device for displaying braille characters. It allows people with visual impairment to access tactile information. It works in combination with the screen reader when connected to a device such as a computer or a smartphone. Versions including a Perkins keyboard also work as a standalone device.

Voice assistants such as iOS's Siri, Android's Google Now, and Microsoft's Cortana are applications that facilitate opening applications and entering text non-manually; however, they have limitations. Some applications require manual login in order to open, actions such as adding new contacts require permissions that must be manually granted. It has been reported that Siri has problems processing long dictations [9]. On the other hand, the results of a study showed that $90 \%$ of people with blind and low vision use dictation on their smartphones, but people with low vision were more satisfied using dictation than those with blindness [4]. Dictation is the fastest way of entering textual information in smartphones, but it is not that efficient even for the slightest text editing tasks.

Transfer devices are assistive technology that allow people to be transferred from one spot to another. For example, people with motor disability who need to move from a bed to a wheelchair. These devices usually use electrical or hydraulic power. There are several variations of wheelchairs such as manual or electrical which can be controlled through a joystick, helmet, sip-and-puff, among others.

Adaptive switches are electronic devices that allow people with motor impairment controlling and operating AT in their environment. Some common examples are joysticks, buddy button, chin buttons, sip-and-puff, among others. Sip-and-puff allows people with motor disabilities to communicate or to control another device by inhaling or exhaling on a tube. This interface sends signals using air pressure by inhaling or exhaling on a tube. It is used for people who can't use their hands like people with quadriplegia.

Wearable devices play an important role in the accessibility for people with motor impairment. They can be anything from small wristbands to backpack computers. Usually, a wearable technology is a combination of several devices. The goals of wearables are three: 1) to be mobile; 2) to augment reality; and 3) to provide context sensitivity [4]. Nowadays, a wearable device has become not only practical, but financially feasible; which means it can be used as an assistive technology to support people with disabilities [8]. In this sense, wearable technology has been offering real opportunities to compensate the loss of mobility for people.

Bracelets as a wearable device have become popular. They allow to read user's physiological variables in an easy and noninvasive way [15]. Likewise, bracelets have been also used to support mobile interaction. Ye et al. [22] designed a wristband prototype to elicit feedback on wearable interaction for people with visual impairment. The Myo band converts the electrical activity in the arm muscles into gestures to control technological devices [21].

Myo armband is a wearable designed as a gesture remote control for the computer, mobile phone, or radio-controlled devices. This device has been largely used by people who need rehabilitation and for game purposes [20]. Its cost is considerably high, and unfortunately, its production has officially ended [17].

Smartphones offer the possibility to connect external input devices through the switch configuration feature: Switch Access in Android [1], and Switch Control in iOS [3]. This feature allows users to interact with their smartphone using hardware switches as an alternative to the touchscreen; this is very helpful for people with motor disabilities when they cannot interact directly with their mobile. Users can connect an external switch, an external keyboard, or use the physical buttons on the Android device or in Apple devices.

However, external devices are not always convenient for portability, since they are not compact and must be carried separately. Likewise, some users with motor disability are not able to press physical buttons.

As can be seen, although there are accessibility features and devices for people with either motor or visual impairment, there is no feasible option for people with both disabilities. Therefore, the purpose of this study is to design hardware solutions in the form of physical switches to facilitate navigation in smartphones to users with these impairments.

\section{User analysis}

We based our design in the characteristics and needs of a user who has both visual and hand-motor impairment. The user is a Mexican female, 50 years old, single. She studied a Bachelor of Business Management, and she recently finished a Master of Human and Organizational Management. From childhood, she gradually lost her sight and became blind at age 39; she has suffered from rheumatoid arthritis for over 30 years, which caused her deformity and severe motor impairment in hands and feet. She walks aided by a quad cane for support, and the help of someone else for guidance. She cannot use the white cane that people with blindness use, because the distortion in her hands makes it difficult to hold it, and because the white cane cannot give her the support she needs due to the motor impairment in her feet. She is able to control the movement of the wrists and the metacarpi, but not of the phalanges; her hand muscles are weak [8]. The user's needs using a smartphone, as well as a detailed description of the difficulties doing them using the screen reader and the voice assistant, are reported in [8]. The needs are not different from those of a user without disability; we can summarize them in: communication activities (chat by text and voice and email), everyday activities (manage agenda, add contacts, set alarm), and work related activities (internet searches, reading contents, note taking) [8]. In our current work we focus on facilitating navigation, which is required in all activities involving a smartphone.

We held an extensive interview with the participant in order to better understand her physical possibilities and limitations relevant to the current proposal. The interview was composed of semi-structured questions, aimed to: 1) understanding the participant's limitations making gestures using the screen reader and the voice assistant, 2) exploring the range of movements on fingers, wrists, elbows and shoulders, and 3) analyzing the participant's use of objects that she finds easy to hold or wear in everyday life activities. The interview lasted approximately 2 hours and it was conducted by two observers. The session was recorded in video. 


\section{Results}

The participant uses an iPhone 5. She cannot hold the phone with one hand, and therefore she places it on a surface such as a table or her lap. She places the phone upside-down because this way it is easier for her to reach the physical button and to do gestures (see Figure 1B, 1C).
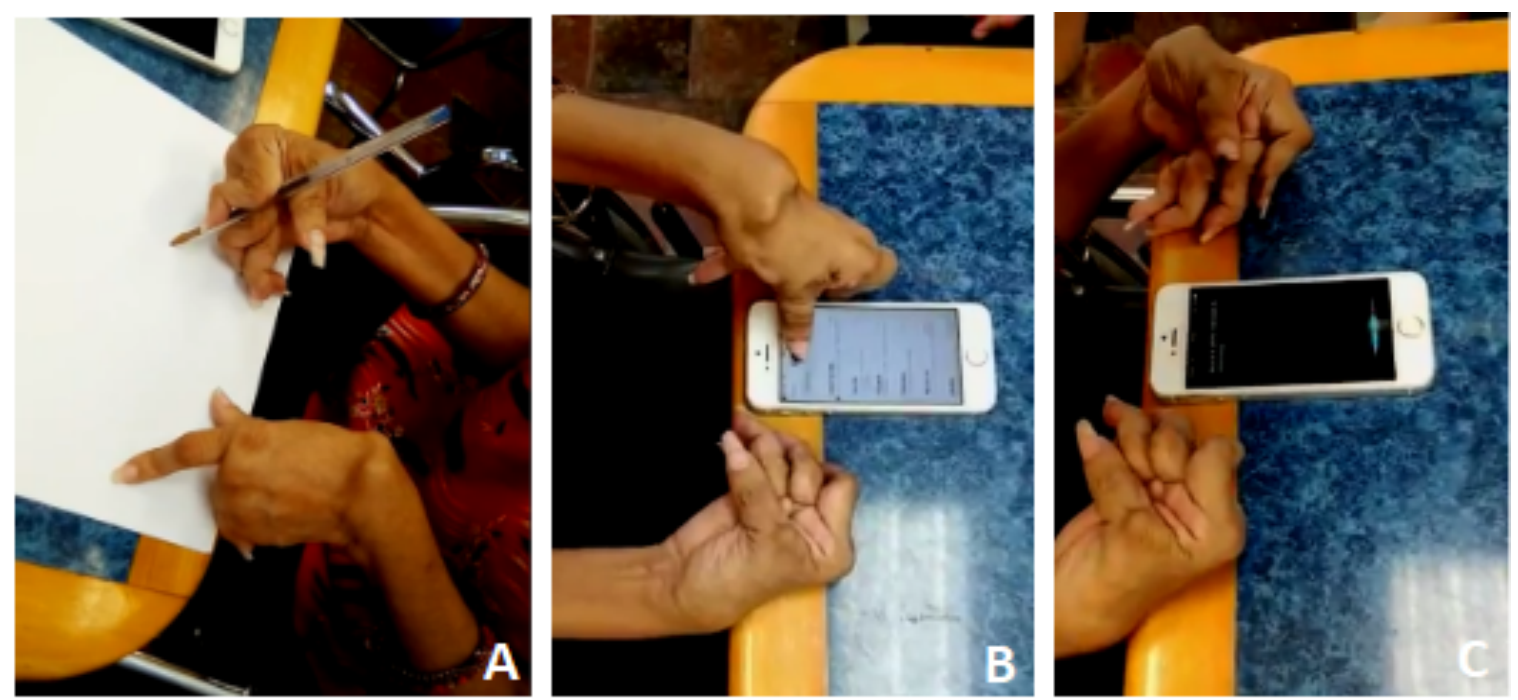

Figure 1. A) Holding a pen, B) Doing gestures, C) Using the voice assistant.

She has no difficulty moving her shoulders up and down, and her wrists are flexible; she is able to move the metacarpi but she cannot exert significant strength. She is able to move her elbows, and she has enough strength to lift them up to an angle of approximately $45^{\circ}$, after which she needs to help herself using the other hand in order to lift the elbow further up for activities such as taking a shower, which she needs to do in a sitting position. Because of the deformity of her hands, she holds a pen with difficulty (see Figure 1A). She finds it easier to hold a pen in upward direction (for example, applying eye-liner) than in downward direction (for example, doing a signature). She puts on makeup and accessories without assistance; for her daily activities she wears earrings, different types of bracelets and hair pins.

The participant does a lot of activities mainly at home and at school, which implies travelling between different places during the day. Because of the motor impairment she cannot carry backpacks, shoulder bags or any bulky or heavy bag, therefore the size and weight of the things she can carry in her purse is critical. On the other hand, it often happens that she misplaces objects and then has a hard time finding them.

\section{Proposed solutions}

Taking into account the physical range of movements, the constraints of the user, the actions she needs to perform on a smartphone, and the context of use, we propose two devices that allow users to do alternative actions to the gestures that users with motor disabilities find difficult doing, such as swiping right and left, tapping and double tapping; these are basic gestures for performing browsing and selection actions such as going left, right and selecting an item. Screen readers enable these actions for linear browsing, and those are also the actions that are usually configured when using Switch Access [1] or Switch Control [3] in order to connect external input devices for people with hand motor disabilities. Both proposals require the use of a screen reader and the switch access to connect with the phone via Bluetooth.

Additionally, the proposed solutions have to be light, compact and portable but not easily misplaced, and of course, they must be comfortable to the user.

\subsection{First Proposal: A Mounted Case}

The first proposal consists of a case to be mounted on top of the smartphone. Since the device is to be used with a screen reader, movements of the finger across the touch surface are not required while browsing; this is because the elements are browsed linearly with swipes to left (next item) and right (previous item). The device consists of four buttons to adapt the following actions (order from top to bottom):

1st: Enter (alternative to double tap)

2nd: Exit (go back)

3rd: Next item (alternative to swipe left)

4th: Previous item (alternative to swipe right)

Buttons measure $1.5 \times 1.5 \mathrm{~cm}$ each, and are located on a single side of the phone for easy access with the thumb; the buttons should be sensitive enough in order to require just a minimum effort pressing them (Figures 2 and 3).

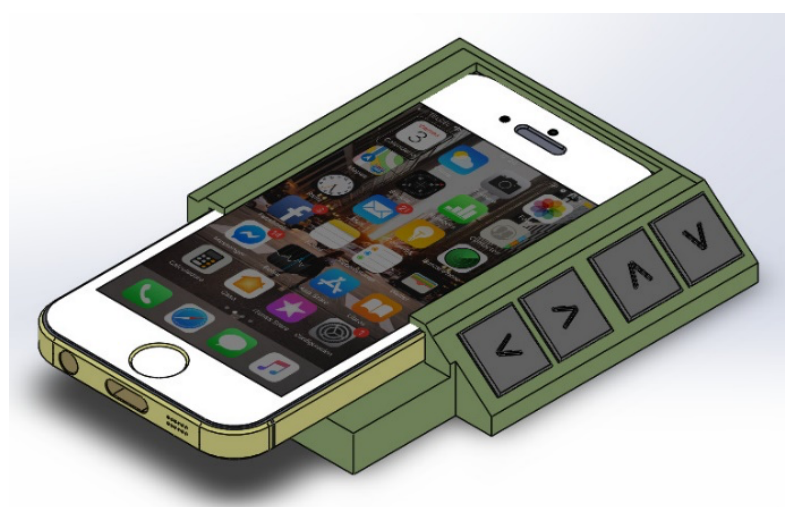

Figure 2. Lateral view of the prototype mounted on the phone. 


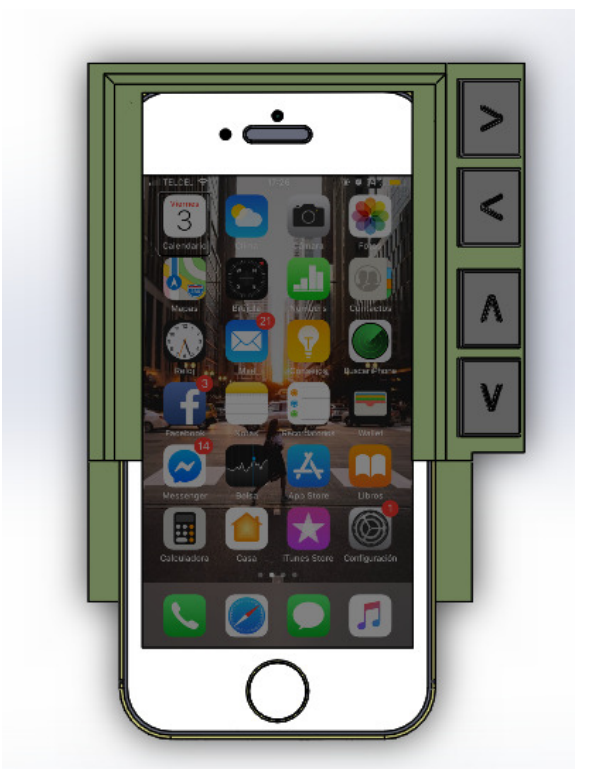

Figure 3. Frontal view of the prototype

This proposal has the following advantages: the case is attached to the device, so it cannot be easily misplaced; it allows for easy actions through soft press on buttons. Unlike physical buttons arranged in different parts of the phone, the buttons in the case are placed close together on one side so that they are located within reach with minimum finger movement; besides, the buttons in the case are bigger than those of the phone. The case can be placed on either end of the phone, and it is designed to facilitate finger movements while using the phone on a surface, which is the way our participant uses it, as well as other people with motor disability who are not able to hold the phone.

The disadvantages of this proposal are that it adds some bulk to the phone, which may be uncomfortable to the user; it needs further design considerations in order to accommodate different phone sizes; even though it can be produced with components that are not too heavy, it represents extra weight on the phone.

Also, in the case of our participant, the upside-down position in which she uses the phone implies that she will reach the buttons from the left side; however, we should not assume that accessing the buttons from the left will be uncomfortable, since the phone will be located on a surface. We will need user testing in order to make further design decision on this matter. On the other hand, we do not have enough evidence from other people with motor disability using the phone in upside-down position, but if it is the case, we would consider this situation in the next design iteration.

From the disadvantages of this first prototype, it could be suggested that an alternative solution would be convenient, this time exploring a different perspective.

\subsection{Second Proposal: An Electronic Bracelet}

Unlike the first proposal, the second one takes advantage of the forearm and elbow movements of the user in an angle range that is within the user's possibilities. The proposal consists of an input device in the form of a bracelet (Figure 4 and 5), and it does not require finger movements or applying pressure on buttons.
Similarly to the first proposal, the actions that the bracelet allows the user to do are: move to the previous element, move to the next element, enter/select, and exit/going back. The bracelet is also designed to be used along with the screen reader, which the user is already familiar with.

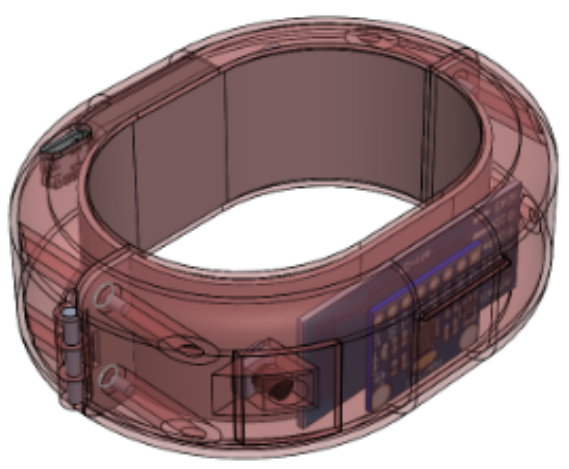

Figure 4. Prototype of the bracelet.

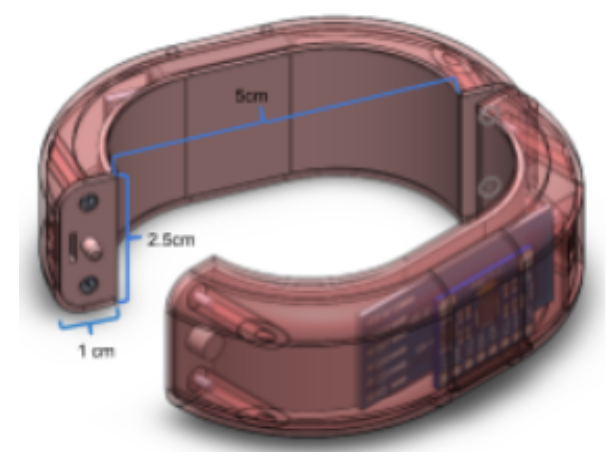

Figure 5. Closing mechanism and dimensions.

The alternative user gestures to perform the actions are shown in the following images. Gesture configuration can be personalized.

Navigation is achieved by doing angular movements with the forearm with respect to the point of origin (coordinates $0,0,0$ ), which is the elbow of the user, in $90^{\circ}$ position between the shoulder and the forearm (Fig. 3A). The user can be standing up or sitting down. Navigating to the previous item requires doing an angular movement of approximately $15^{\circ}$, to the left (Fig. 3B); navigating to the next item requires moving the forearm approximately $15^{\circ}$ to the right (Fig 3C).

In order to do an Enter action, equivalent to a tap or double tap on an item, it is necessary to move the forearm approximately $5 \mathrm{~cm}$ forward, opening the elbow angle (Fig. 3D); and lastly, the exit or back action is performed by moving the forearm approximately $5 \mathrm{~cm}$ backward, closing the elbow angle (Fig. 3E).

The design allows the user to wear and take off the bracelet without assistance; once worn, the bracelet does not require the user to remember its location. Though considered as an option at the beginning, we discarded the possibility of designing a pointing device in the shape of a pen because it could be difficult to hold, it would require applying pressure, and it could be easily misplaced. 

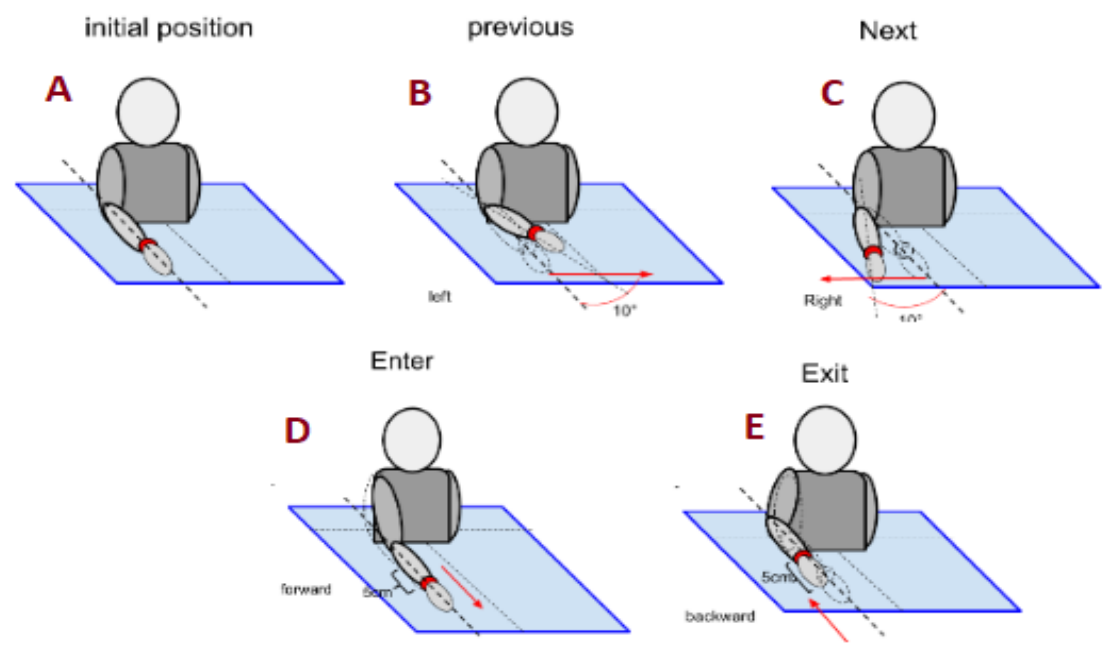

Figure 6. Gestures that a user can do with the bracelet.

The bracelet holds itself closed using a magnet, and it gets opened by pulling any of the edges. On the upper surface it has a relief indicating the correct orientation of the bracelet, similar to the one located in keyboards to indicate the position of the keys by touch. It has an ON/OFF switch for saving battery, and it can be charged via micro USB while not worn. Its dimensions are $5 \mathrm{~cm}$ wide, $2.5 \mathrm{~cm}$ tall and $1 \mathrm{~cm}$ thick. Internally, the bracelet is composed of an accelerometer Mpu6050, an Arduino Pro Mini that is a microcontroller board based on the ATmega328, and a Bluetooth module $\mathrm{HC}-05$ for interpreting, processing and sending the signals to the smartphone. It has a LiPo $3.7 \mathrm{v}$ rechargeable battery. The estimated cost of the prototype is 60 USD.

The advantages of this second proposal are that the device is used separately from the phone, thus avoiding extra bulk on it, but adding the weight and bulk to the wrist; on the other hand, since it is a wearable device it might not feel like an extra bulk, because it can be considered as an accessory as others that she already uses in everyday life; another advantage is that it is not easy to be misplaced while using it, because it is meant to be worn by the user. On the other hand, using the bracelet does not represent any extra design consideration when the user places the phone in an inverted position, as it is the case of our participant.

The proposal has the following disadvantages: derived from the use of an accelerometer, difficulties arise when the user is in motion, for example when using the phone while in a car, which is a very common scenario of use; because of this, the device requires careful programming to preview both the scenarios when the user stands still and when (s)he is moving around; on the other hand, not all people might feel comfortable using a bracelet.

Table 1 presents a summary of the characteristics of both proposals.

Table 1. Summary of both proposals.

\begin{tabular}{|l|c|c|}
\hline \multicolumn{1}{|c|}{ Feature } & Case & Bracelet \\
\hline Base of movement & Finger (thumb) & Elbow \\
\hline Mount on & Phone & Wrist \\
\hline Connection with & Bluetooth & Bluetooth \\
\hline
\end{tabular}

\begin{tabular}{|l|c|c|}
\hline \multicolumn{1}{|c|}{ Feature } & Case & Bracelet \\
\hline phone & & \\
\hline Use with & $\begin{array}{c}\text { Screen reader, } \\
\text { switch access }\end{array}$ & $\begin{array}{c}\text { Screen reader, } \\
\text { switch access }\end{array}$ \\
\hline Weight & $\begin{array}{c}100-130 g r \\
\text { (estimated) }\end{array}$ & $\begin{array}{c}100-130 g r \\
\text { (estimated) }\end{array}$ \\
\hline
\end{tabular}

In both proposals, the device is meant to behave as controller of the Switch Access or Switch Control, by detecting the user's interactions and transmitting them to the smartphone via Bluetooth; the smartphone must in turn be configured to receive the different events representing the usual gestures. The estimated weight of either device is between 100 and 130 gr.

\section{Conclusions and future work}

Smartphone users with disabilities still have access issues. Wearable devices represent an opportunity to compensate for the loss of mobility and sight for people. In this paper, we presented an analysis of the physical characteristics of a user with both visual and motor impairment, as well as the user's context of smartphone use. We also presented two proposals of physical switches aiming to alleviate the access difficulties of this user type, in particular by facilitating navigation through gestures that they find easy to do. It is important to mention that not all types of motor disability are the same, therefore these proposals are aimed to users with physical characteristics similar to those of the participant of our study.

As future work, user intervention is necessary in order to decide which one of the proposals would be more convenient, and to make the necessary improvements to either one. User input is also necessary in order to make decisions about the material to use for building the device, and to measure its usability.

\section{Acknowledgments}

We want to thank the participant for her willingness to help us carrying out this research. Thank you for giving us your precious time; we hope that our proposals will improve your use of smartphones in the near future. 


\section{References}

[1] Android Accessibility Help. About Switch Access for Android.https://support.google.com/accessibility/android/ans wer/6122836

[2] Anthony, L., Kim, Y., and Findlater, L. Analyzing usergenerated youtube videos to understand touchscreen use by people with motor impairments. In Proc. CHI 2013, ACM Press (2013), 1223-1232. DOI: 10.1145/2470654.2466158.

[3] Apple Support. Use Switch Control to navigate your iPhone, iPad, or iPod touch. https://support.apple.com/enus/HT201370

[4] Azenkot, S., and Lee, N. Exploring the use of speech input by blind people on mobile devices. In Proceedings of the 15th International ACM SIGACCESS ASSETS, ACM Press (2013) http://dx.doi.org/10.1145/2513383.2513440

[5] Billinghurst, M., and Starner, T. Wearable Devices: New Ways to Manage Information. Computer 32, 1 (1999), 57-64. DOI=http://dx.doi.org/10.1109/2.738305

[6] Carvalho, L., Melchiori, B., Santos, F., Pereira, L., and Pimenta Freire, A. Accessible smart cities?: Inspecting the accessibility of Brazilian municipalities' mobile applications. In Proc. of the 15th Brazilian Symposium on Human Factors in Computing Systems 2016, ACM Press. DOI: https://doi.org/10.1145/3033701.3033718

[7] Chiti, S., and Leporini, B. Accessibility of Android-based Mobile Devices: A Prototype to Investigate Interaction with Blind Users. In Proc. of the 13th International Conference on Computers Helping People with Special Needs, SpringerVerlag, Berlin, Heidelberg (2012), 607-614. DOI: http://dx.doi.org/10.1007/978-3-642-31534-3_89

[8] Dibia, V., Trewin, S., Ashoori, M., and Erickson, T. Exploring the Potential of Wearables to Support Employment for People with Mild Cognitive Impairment. In Proc of the 17th International ACM SIGACCESS, ACM Press (2015), 401-402. DOI: 10.1145/2700648.2811390

[9] Fajardo-Flores, S. B., Gaytán-Lugo, L. S., Santana-Mancilla, P. C., and Rodríguez-Ortiz, M. A. Mobile Accessibility for People with Combined Visual and Motor Impairment: A Case Study. In Proc. of the 8th CLIHC, ACM Press (2017). https://doi.org/10.1145/3151470.3151476

[10] Findlater, L., Moffatt, K., Froehlich, J. E., Malu, M., and Zhang, J. Comparing Touchscreen and Mouse Input Performance by People With and Without Upper Body Motor Impairments. In Proc. CHI 2017, ACM Press (2017), 6056-6061. DOI: https://doi.org/10.1145/3025453.3025603

[11] Grussenmeyer, W., and Folmer, E. Accessible Touchscreen Technology for People with Visual Impairments: A Survey. ACM Trans. Access. Comput. 9, 2, (2017). DOI: https://doi.org/10.1145/3022701

[12] Illinois Library. Blind/Visual Impairment: Common Assistive Technologies.
[13] Kane, S. K., Jayant, C., Wobbrock, J. O., and Ladner, R. E. Freedom to roam: a study of mobile device adoption and accessibility for people with visual and motor disabilities. In Proc. of the 11th international ACM SIGACCESS, ACM Press (2009), 115-122. DOI: https://doi.org/10.1145/1639642.1639663

[14] Leporini, B. Buzzi, M. C., and Buzzi, M. Interacting with mobile devices via VoiceOver: usability and accessibility issues. In Proc. OzCHI 2012, ACM Press (2012), 339-348. DOI: http://dx.doi.org/10.1145/2414536.2414591

[15] Moore, S. A., Da Silva, R., Balaam, M., Brkic, L., Jackson, D., Jamieson, D., Ploetz, T., Rodgers, H., Shaw, L., van Wijck, F., and Price, C. Wristband Accelerometers to motiVate arm Exercise after Stroke (WAVES): study protocol for a pilot randomized controlled trial. Trials 17, 1 (2016). DOI: https://doi.org/10.1186/s13063-016-1628-2

[16] Naftali, M., and Findlater, L. Accessibility in Context: Understanding the Truly Mobile Experience of Smartphone Users with Motor Impairments. In Proc. of ASSETS 2014, ACM Press (2014) http://dx.doi.org/10.1145/2661334.2661372

[17] North. Getting starting with Myo on Windows. https://support.getmyo.com/hc/en-us/articles/202657596

[18] Paim, P., and Prietch, S. Problems and Solutions in the Design for Deaf Persons who are Sign Language Users to Adopt Assistive Technology Products. Journal on Interactive Systems 10, 2 (2019), 70-81. DOI https://doi.org/10.5753/jis.2019.554

[19] Pielot, M., Kazakova, A., Hesselmann, T., Heuten, W., and Boll, S. PocketMenu: non-visual menus for touch screen devices. In Proc. of the 14th international conference on Human-computer interaction with mobile devices and services, ACM Press (2012), 327-330. DOI: https://doi.org/10.1145/2371574.2371624

[20] Sadeghi Esfahlani, S., Muresan, B., Sanaei, A., and Wilson, G. Validity of the Kinect and Myo armband in a serious game for assessing upper limb movement. Entertainment Computing 27, (2018), 150-156. DOI: https://doi.org/10.1016/j.entcom.2018.05.003

[21] Qamar, A. M., Riaz Khan, A., Osama Husain, S., Abdur Rahman, M., and Baslamah, S. A Multi-Sensory GestureBased Occupational Therapy Environment for Controlling Home Appliances. In Proc. of the 5th ACM on International Conference on Multimedia Retrieval, ACM Press (2015), 671-674. DOI: http://dx.doi.org/10.1145/2671188.2749412

[22] Ye, H. Malu, M., Oh, U., and Findlater, L. Current and future mobile and wearable device use by people with visual impairments. In Proc. CHI 2014, ACM Press (2014), 3123 3132. DOI: https://doi.org/10.1145/2556288.2557085

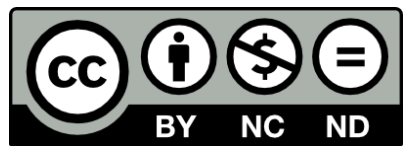

(C) 2020 by the authors. This work is licensed under the Creative Commons AttributionNonCommercial-NoDerivatives 4.0 International License. To view a copy of this license, visit http://creativecommons.org/licenses/by-nc-nd/4.0/ or send a letter to Creative Commons, PO Box 1866, Mountain View, CA 94042, USA. 